\title{
Bladder Cancer Diagnosis using Artificial Neural Network
}

\author{
Shaymaa M. \\ AlKashef \\ Com. Eng. \& \\ systems Dept. \\ Mansoura University \\ Mansoura, Egypt
}

\author{
Abdelhameed \\ Ibrahim \\ Com. Eng. \& \\ systems Dept. \\ Mansoura University \\ Mansoura, Egypt
}

\author{
Hesham Arafat \\ Com. Eng. \& \\ systems Dept. \\ Mansoura University \\ Mansoura, Egypt
}

\author{
Tarek A. El- \\ Diasty \\ Urology \& \\ Nephrology Cen., \\ Mansoura University \\ Mansoura, Egypt
}

\begin{abstract}
The analysis of Magnetic Resonance Imaging (MRI) images using Artificial Neural Network (ANN)-based system is implemented in this paper to achieve a rapid and accurate diagnosis tool for bladder cancer. The proposed approach comprises image enhancement, removal of border, feature extraction and bladder cancer recognition using multilayer perception (MLP) with sequential weight/bias training function. We develop a model that defines the cancer level in order to enhance its treatment. Experimental results show that the devised approach increases the accuracy of diagnosis of bladder cancer up to $95 \%$.
\end{abstract}

\section{General Terms}

Pattern Recognition and Image Processing.

\section{Keywords}

Bladder cancer, Image segmentation and ANN.

\section{INTRODUCTION}

The bladder cancer is potentially a very serious condition that can be life threatening $[1,2]$. It is the fourth most common cancer among men, and the eighth most common cause of cancer among women [3]. It has an average age at diagnosis of 65 years. Consequently, its early detection is vital in increasing the chances of successful treatment. Bladder cancer staging is a multifaceted process that utilizes a combination of clinical and radiological assessment to evaluate the degree of disease spread [4].

A variety of clinical diagnostic and therapeutic techniques for bladder cancer have been developed to improve the quality of the diagnostic methods [5]. In the past, computed tomography (CT) has been used to stage bladder tumors, but dynamic contrast-enhanced magnetic resonance imaging (MRI) has been shown to be superior to CT for this purpose [6]. Thereby, the aim of the present work is to contribute to the improvement of biomedical systems, allowing for a noninvasive diagnosis of bladder cancerous and pre-cancerous tissues.

We use artificial intelligence techniques to early detect bladder cancer and determine tumor staging using a set of functional MRI images. The accuracy of MRI in T staging bladder cancer is assessed. The MRI scans contain different types of information relating to specific $3 \mathrm{D}$ voxels in the bladder. We combine useful information within a particular 3D cell (3D volume in the bladder scan space) from all MRI modalities in an automatic way in order to detect or suspect the cancer in the $3 \mathrm{D}$ cell [7].
The paper is organized as follows. Section II reviews the basic techniques of medical imaging analysis and the artificial neural networks (ANNs) [8]. The magnetic resonance imaging (MRI) is addressed as a medical imaging technique used in radiology. In Section III, the problem to be inspected is defined. The proposed technique is discussed in Section IV. The obtained results are presented and discussed in Section V. Finally, the conclusion is outlined in Section VI.

\section{BASIC TECHNIQUES}

Medical imaging is used to create images of the human organs for clinical purposes to diagnose, examine diseases and study of normal anatomy and physiology [9]. The analysis of the medical image can help in the detection and diagnosis of diseases. Most of clinical equipment can be hardly imagined without video, laser jets and mathematical treatment. Magnetic resonance imaging (MRI) is a medical imaging technique used in radiology to visualize internal structures of the body in detail. The principle of operation is based on the fact that body tissue contains lots of water (and hence protons) which gets aligned in a large magnetic field [10]. Each water molecule has two hydrogen nuclei or protons. When a person is positioned inside the powerful magnetic field of the scanner, the average magnetic moment of many protons becomes aligned with the direction of the field. A radio frequency transmitter is briefly turned on, producing a varying electromagnetic field [11]. The images of bladder cancer are taken with a MRI device shown in Fig 1.

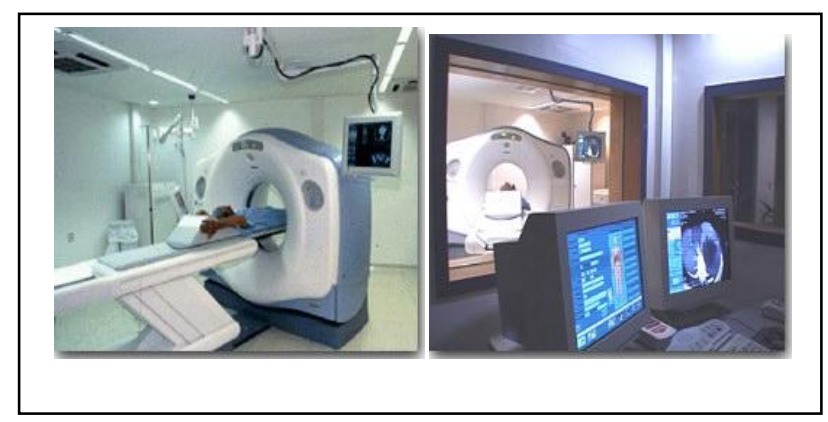

Fig 1: MRI device for bladder cancer images. 
The electromagnetic field with the resonance frequency is absorbed and then flips the spin of the protons in the magnetic field. After the electromagnetic field is turned off, the spins of the protons return to thermodynamic equilibrium and the bulk magnetization becomes re-aligned with the static magnetic field. During this relaxation, a radio frequency signal is generated, which can be measured with receiver coils. Clinically, the bladder cancer can be recognized into different stages as depicted in Fig 2.

The artificial neural networks (ANNs) are simplified models of the biological nervous system. Their performance as a computational model is derived from the way that attempts to emulate the architecture and function of the human brain [12]. The ANN is an information-processing system that has certain performance characteristics in common with biological neural networks [13]. It is a massively parallel distributed processing system made up of highly interconnected neural computing elements that vase the ability to learn and thereby acquire knowledge and make it available for use. The neural networks are applied in various medical applications within the context of advances in medical decision support arising from parallel developments in statistics and artificial intelligence [14]. While ANN methods can be used in several medical domains, applications of particular interest are in the detection of bladder cancer.

An ANN consists of some nodes that are connected via weights. Each node receives data from behind nodes, adds it and passes data through a nonlinear function, and then propagates data to proceeding nodes. Data is processed in discrete "elements" or "neurons" and transferred amongst these elements through "connections" that simulate neural synapses. Artificial neurons or processing elements as the basic components of an ANN can be built in hardware, as specialized "neuro-chips", or modeled in software [15]. They transform the input numerical data, through the use of various mathematical functions, into numerical outputs. The output of each neuron is presented as "connection strength" to all the neurons of the next neuronal layer as depicted in Fig 3. In this work, we use MRI imaging in addition to ANN-based system in order to achieve a rapid and accurate diagnosis tool for bladder cancer.

\section{PROBLEM DEFINITION}

There are different types of information contained in the MRI scans relating to specific $3 \mathrm{D}$ voxels in the bladder. We aim to combine automatically useful information within a particular 3D cell from all MRI modalities in order to detect or suspect that cancer in the $3 \mathrm{D}$ cell. The main challenges can be specified as follows. The first is the alignment problem in the sense that all the modalities have different 3D resolution and orientation. Furthermore, the patient may have moved slightly between the acquisition of one modality and the next. The second is the feature extraction. A large number of information can be present in a specific 3D cell. However, which information is useful for detecting cancer and separating it from normal tissue and tissue from other disorders. The third is the normalization of features; once we identify the useful features, how should they be normalized. The fourth challenge is the process of acquiring ground truth. What should we consider as a reliable ground truth so that we can measure the performance of the system. Finally, there is a detection problem; What values of the features in the $3 \mathrm{D}$ cell that indicate the cancer in the tissue.
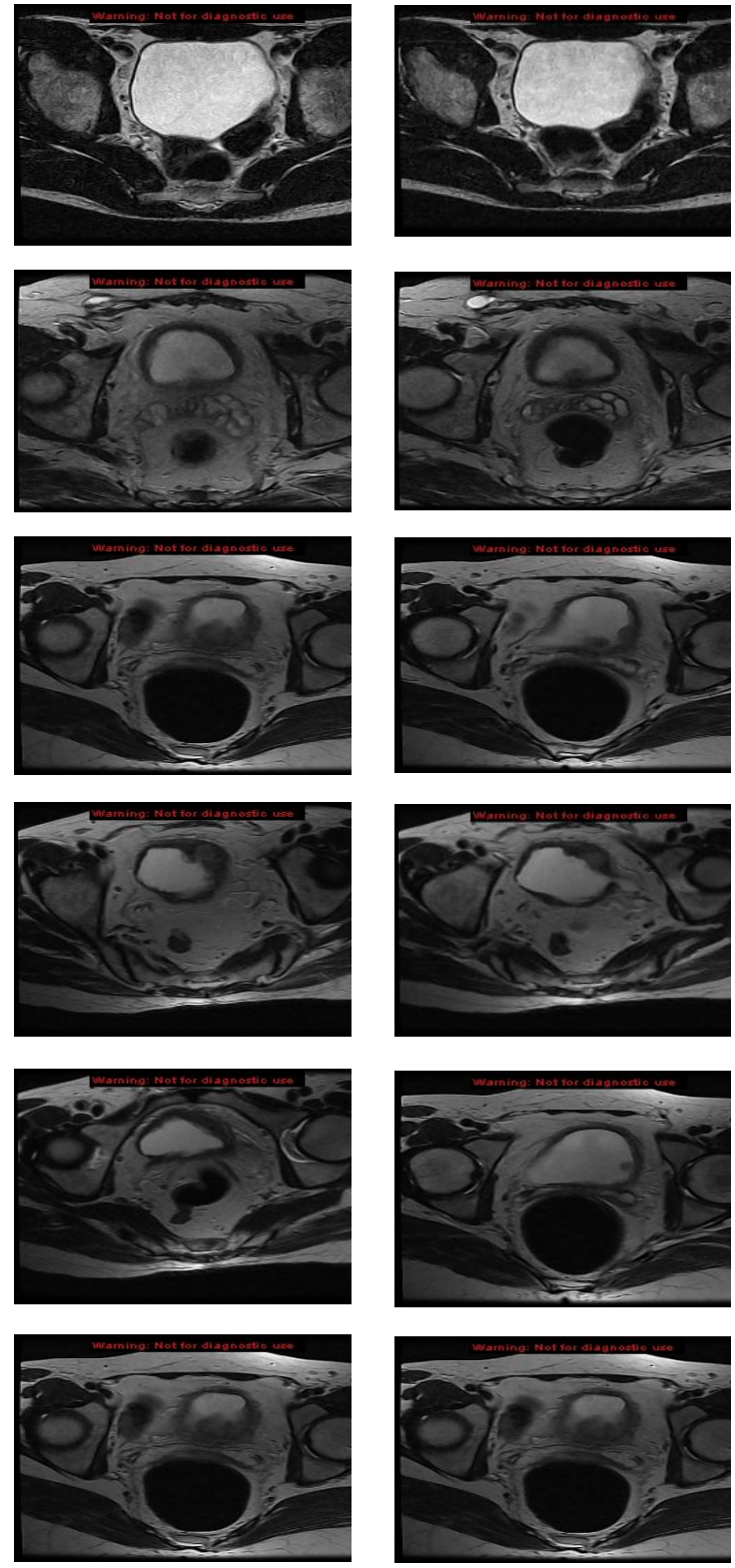

Fig 2: Stages of urinary bladder cancer.

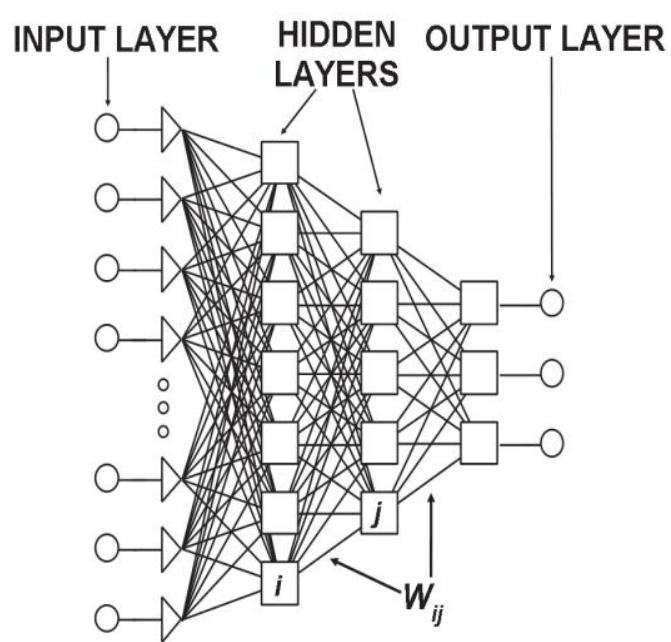

Fig 3: Simplified structure of a neural network. 


\section{PROPOSED TECHNIQUE}

The proposed algorithm, as depicted in Fig 4, comprises four steps; these are the image enhancement, removal of border, feature extraction using DWT features with different mother functions and reduction using PCA, and bladder cancer recognition using MLP with sequential weight/bias training function. In the following, each step is briefly clarified.

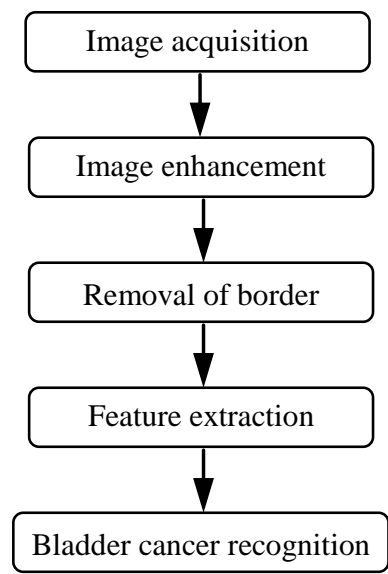

Fig 4: Steps of the proposed algorithm.

\subsection{Image acquisition}

The images of bladder cancer were taken with a MRI device. On average, there are about 40 images for every patient. They were stored in color JPEG format. Matlab is used to convert the color JPEG images into gray scale raw format on the PC.

We use MRI images taken from one angle for 40 patient and we take about 40 images for each patients in different levels. Images are divided into 4 levels or layers .each level contain images from different type of disease (free, T1, T2, T3, T4).

Database images are taken from Urology and Nephrology Center, Mansoura University. We found that when discarding first and last $20 \%$ of images from each level, this would enhance classifier performance.

\subsection{Image enhancement}

The wiener filter is used to convert the input image to the desired output image in such a way that is as best as possible [16]. The best-first solution is considered to be the one that satisfies the minimum sums of the squares of the errors, while the error refers to the difference between a measured value and the calculated value, i.e., the least-squares method. This approach removes white noise from the data. The inverse filtering is a restoration technique for deconvolution. When the image is blurred by known low pass filter, it is possible to recover the image by inverse filtering or generalized inverse filtering. However, inverse filtering is very sensitive to additive noise. The approach of reducing degradation at a time allows us to develop a restoration algorithm for each type of degradation and simply combine them. The wiener filtering

satisfies the minimum sums of the squares of the errors, while the error refers to the difference between a measured value and the calculated value, i.e., the least-squares method. This approach removes white noise from the data. The inverse filtering is a restoration technique for deconvolution. When the image is blurred by known low pass filter, it is possible to recover the image by inverse filtering or generalized inverse filtering. However, inverse filtering is very sensitive to additive noise. The approach of reducing degradation at a time allows us to develop a restoration algorithm for each type of degradation and simply combine them. The wiener filtering executes an optimal trade-off between inverse filtering and noise smoothing. It removes the additive noise and inverts the blurring simultaneously. The wiener filtering is optimal in terms of the mean square error as seen in Fig 5.

\subsection{Removal of Border and Background}

The proposed system uses an algorithm for border and background removal to give the result as shown in Fig 6 .

\subsection{Features Extraction}

Feature extraction is the transformation of the original data (using all variables) to a dataset with a reduced number of variables. In the problem of feature selection, the aim is to select those variables that contain the most discriminatory information. Alternatively, we may wish to limit the number of measurements we make, perhaps on grounds of cost, or we may want to remove redundant or irrelevant information to obtain a less complex classifier.

In feature extraction, all variables are used and the data are transformed (using a linear or nonlinear transformation) to a reduced dimension space. Thus, the aim is to replace the original variables by a smaller set of underlying variables. There are several reasons for performing feature extraction. These include the bandwidth reduction of input data and the provision of a relevant set of features for a classifier, resulting in improved performance, particularly from simple classifiers. Further reasons are reducing redundancy and recovery of new meaningful underlying variables or features so that the data may easily be viewed and relationships as well as the structure in the data can be identified .Only horizontal wavelet features gives us best results.

Wavelets have been demonstrated to give quality representations of images. The discrete wavelet transform (DWT) presents a multiresolution analysis in the form of coefficient matrices which can be used in a manner similar to Fourier series coefficients. This DWT representation can be thought of as a form of "feature extraction" on the original image.

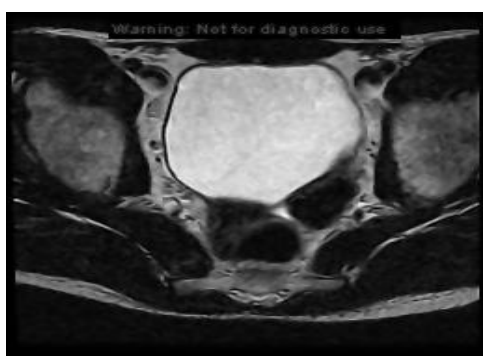

Fig. 5: The image after wiener filter.
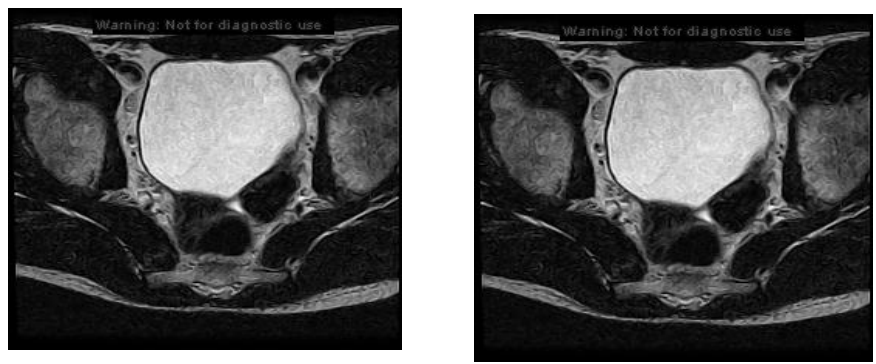

Fig. 6: (a) Original image, (b) Image after removing border and background. 


\subsection{Principal Component Analysis}

Principal components analysis (PCA) [17] derives new variables in decreasing order of importance that are linear uncorrelated combinations of the original variables. Geometrically, principal components analysis can be thought of as a rotation of the axes of the original coordinate system to a new set of orthogonal axes that are ordered in terms of the amount of variation of the original data they account [18].

In some situations, the dimension of the input vector is large, but the components of the vectors are highly correlated (redundant). It is useful in this situation to reduce the dimension of the input vectors. An effective procedure for performing this operation is principal component analysis [19]. This technique has three effects: (i) it orthogonalizes the components of the input vectors so that they are uncorrelated with each other, (ii)it orders the resulting orthogonal principal components so that those with the largest variation come first, (iii) and it eliminates those components that contribute the least to the variation in the data set as depicted in Fig 7 and Fig 8. About 130 principal components gave us best results for each image.

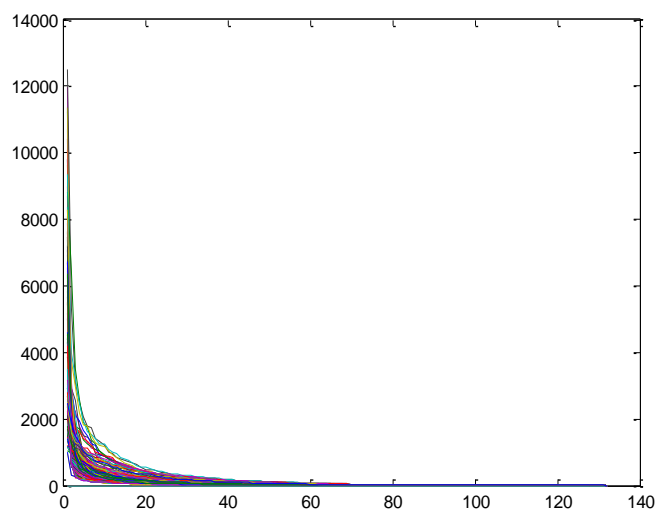

Fig 7: PCA for all images.

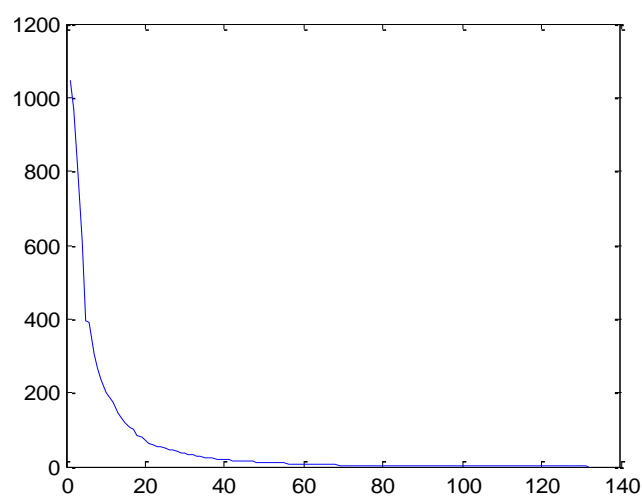

Fig 8: PCA for one image.

\section{EXPERIMENTAL TEST BED}

In the training phase, the neural network is trained with 25 different MRI images with known stages. The training takes about 9 sec. Three trials have been carried out to accomplish best accurately results. In the first trial, 3D images for 40 patients are collected in one folder for testing using MATLAB and the ANN is built to identify the stage of the cancer. Good results were obtained which stimulate carrying out the second modification. In the second testing, we rely only on one section of 40 axial images per patient. The ANN is built for testing the images through image segmentation. Unfortunately, poor results were obtained. In the forth experiment, the 40 images are classified according to the body size, age and sex of the patients into 4 levels. The first level refers to those pre-cancer images, the second and third levels comprises cancer-infected images with high and low rates, and the fourth level contains cancer-free images. The images of each level is collected in a specific folder. In order to attain the best performance, the ANN is trained using 40 images per patient with information of 40 patients. However, the targeted results were not obtained. Consequently, we modify our experiment by excluding the cancer-free parts and estimating the mother function in conjunction with the percentage of the excluded parts until getting the highest performance and achieving the best results.

\section{RESULTS}

A multilayer perception (MLP) is a feed forward artificial neural network model that maps sets of input data onto a set of appropriate outputs. An MLP consists of multiple layers of nodes in a directed graph, with each layer fully connected to the next one. Except for the input nodes, each node is a neuron (or processing element) with a nonlinear activation function. MLP utilizes a learning technique called back propagation for training the network. MLP is a modification of the standard linear perception and can distinguish data that are not linearly separable.

MLP consists of three layers :input layer have about 130 neuron ,hidden layer have 100 neuron, output layer have 1 neuron

In the training phase, the neural network is trained with 25 different MRI images with known stages. The training takes about 9 sec. Figures 9-11 show neural network training interface, training curve, and regression for level 1 image when using DB mother function level 5. The neural network training interface, training curve, and regression for level 2 image when using DB mother function level 5 are depicted in Figs. 12-14. Figures 15-17 show neural network training interface, training curve, and regression for level 3 image when using DB mother function level 5. The neural network training interface, training curve, and regression for level 2 image when using DB mother function level 5 are depicted in Figs. 18-20.

\section{CONCLUSION}

An intelligent method was developed for classifying bladder cancers to specific diagnostic categories based on cancer stage (FREE, T1, T2, T3, T4) using ANN and MRI. The ANN was trained using the 40 patient MRI image. These images were divided into four categories according to the layer. The ANNs correctly classified all samples and identified the images most relevant to the classification. Sequential weight/bias training algorithm was chosen as learning function because of its speed and accuracy. DWT was chosen as a feature extraction technique with only horizontal features to achieve the best results. About 130 PCA features were introduced as input to ANN. We achieve accuracy rate of $92 \%$ for our database. To test the ability of the trained ANN models to recognize MRI bladder cancer images, we analyzed additional blinded samples. The obtained results dictates the importance and validly of the devised approach as its accuracy increases from the currently $70 \%$ achieved by Urology and Nephrology Center, Mansoura University up to $95 \%$. 


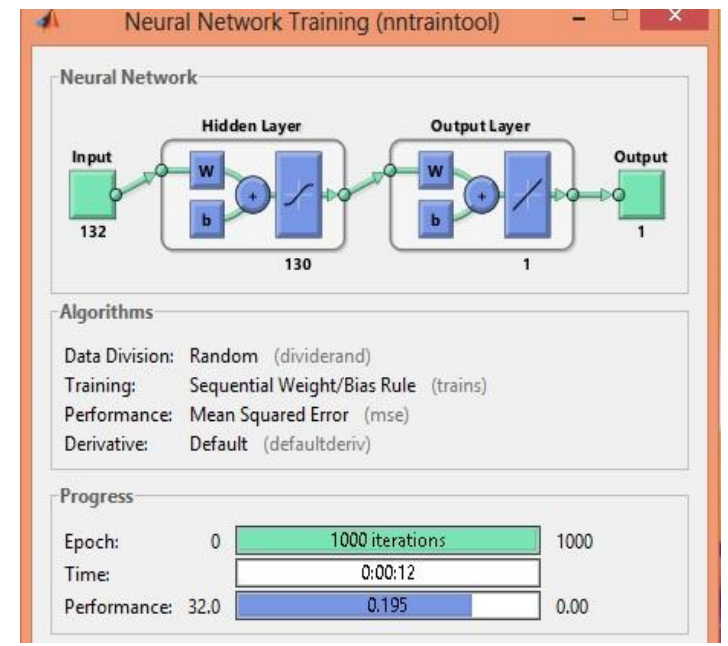

Fig. 9: Neural network training interface for level 1.
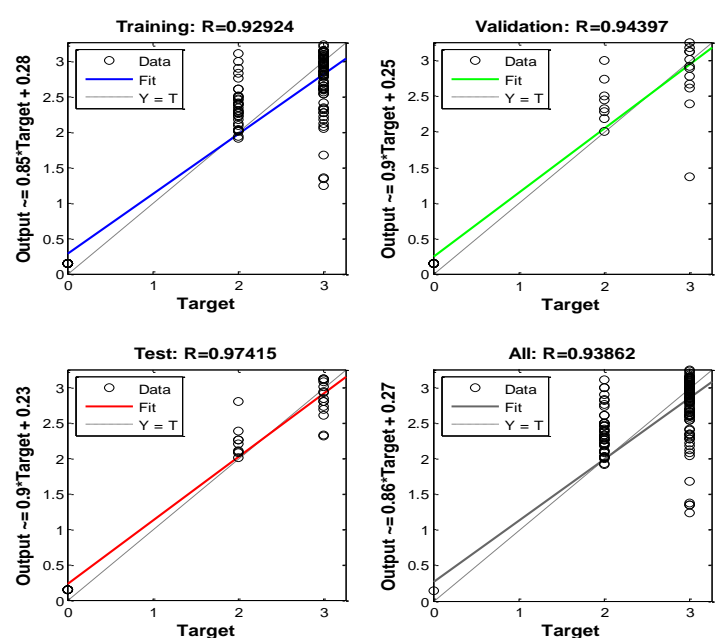

Fig 10: Neural network training curve for level 1.

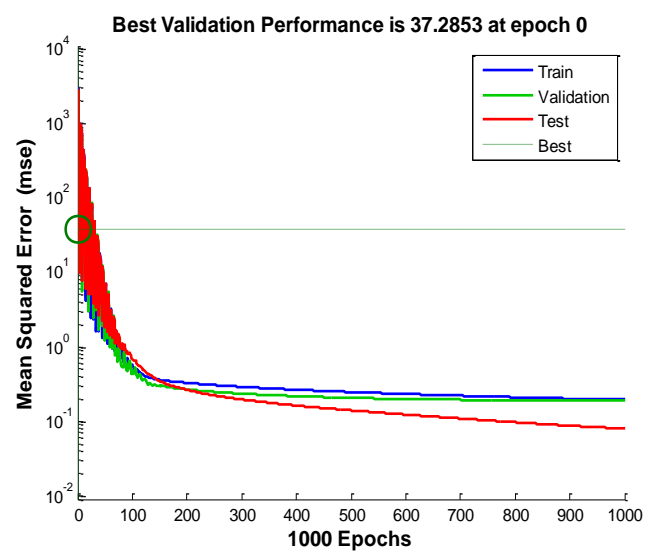

Fig 11: regression for level 1 image when using DB mother function level 5.

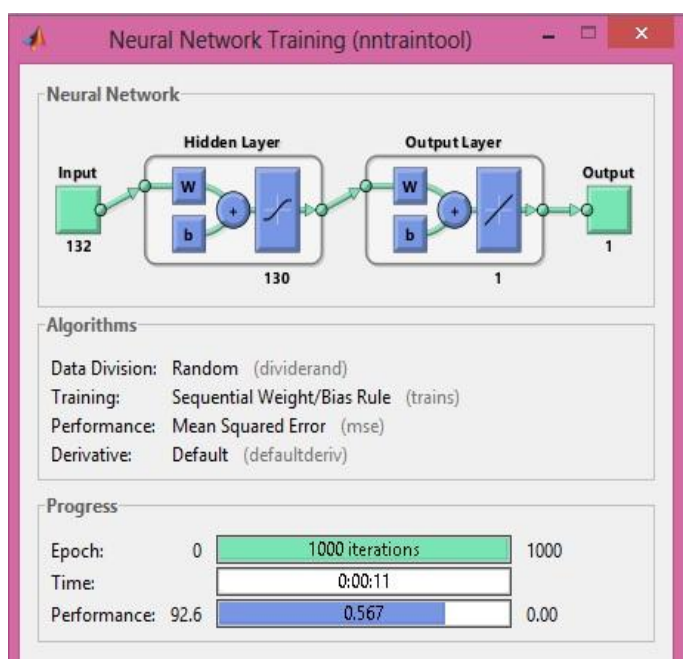

Fig. 12: Neural network training interface for level 2.

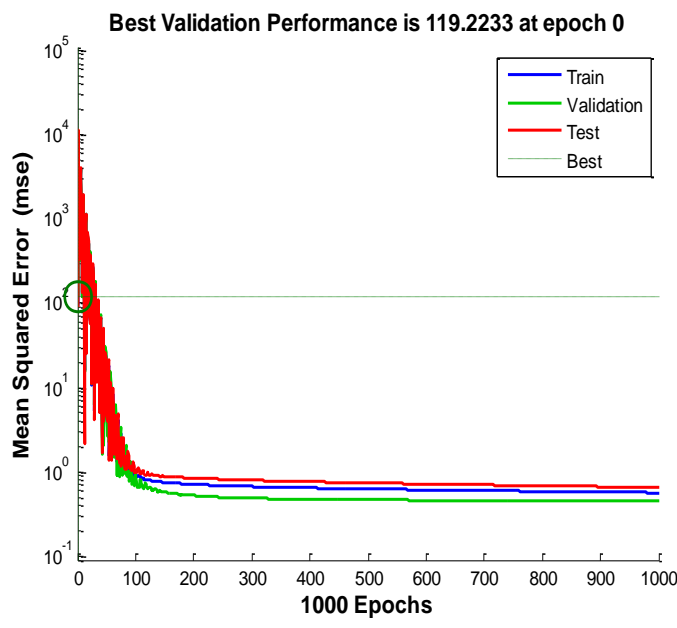

Fig. 13: Neural network training curve for level 2.
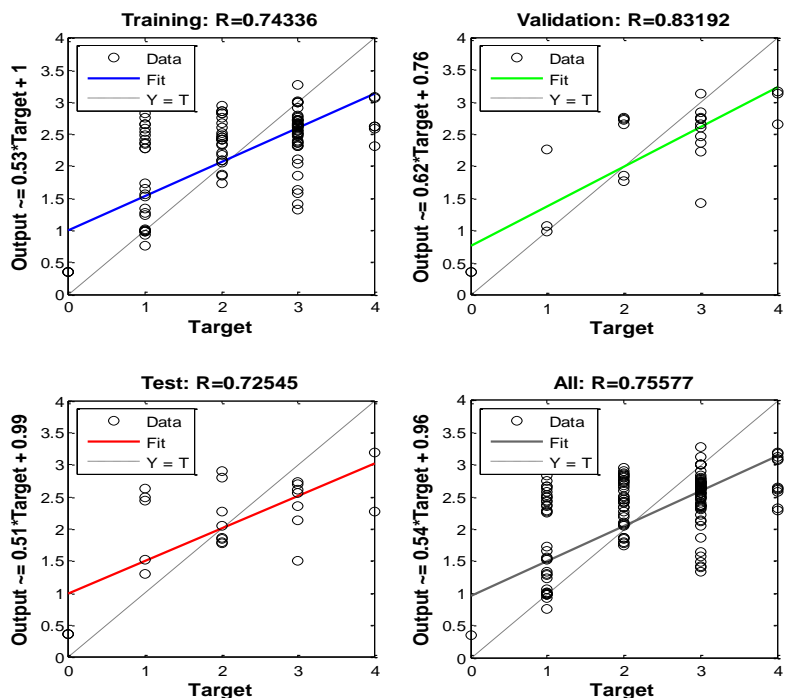

Fig. 14: regression for level 2 image when using DB mother function level 5. 


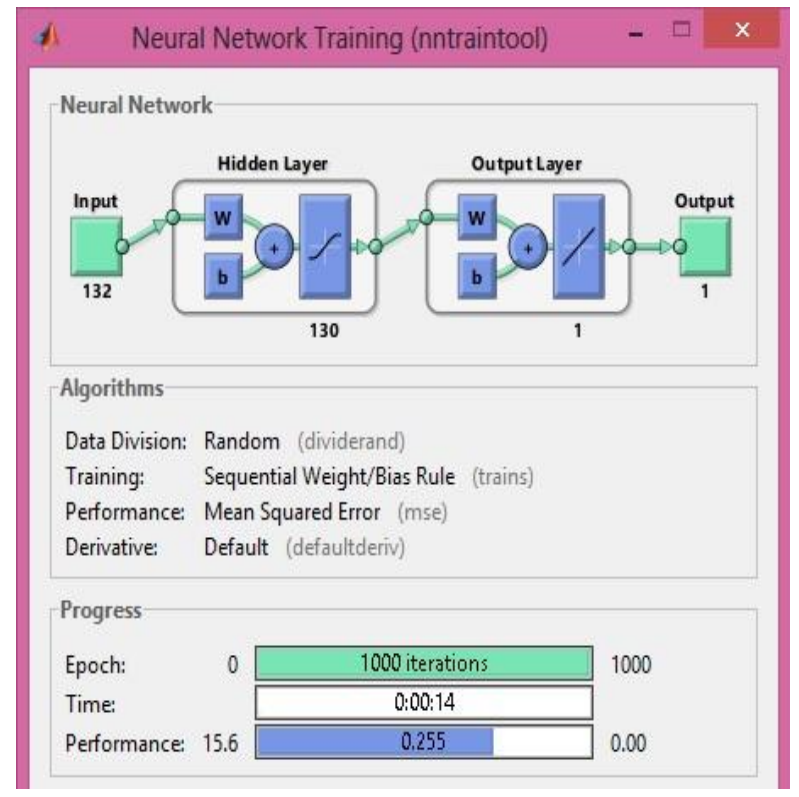

Fig 15: Neural network training interface for level 3.

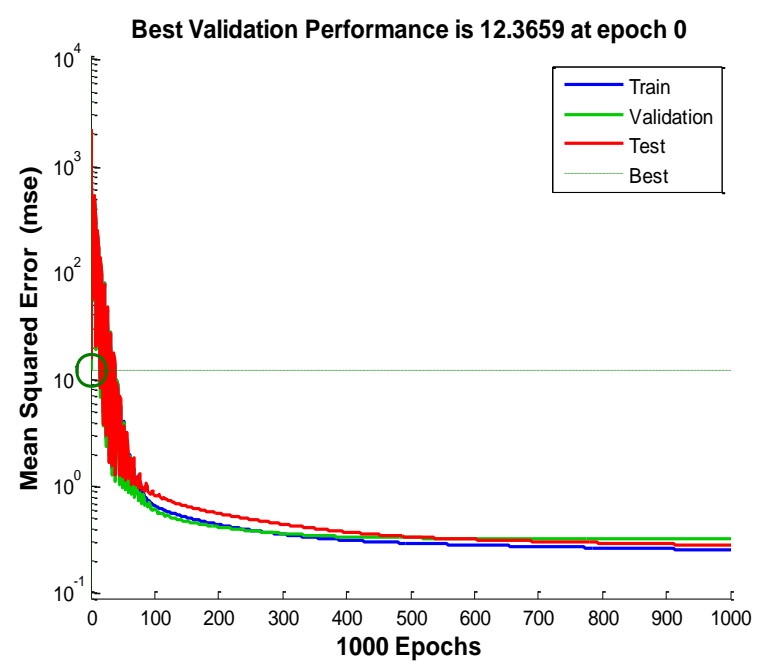

Fig 16: Neural network training curve for level

3.
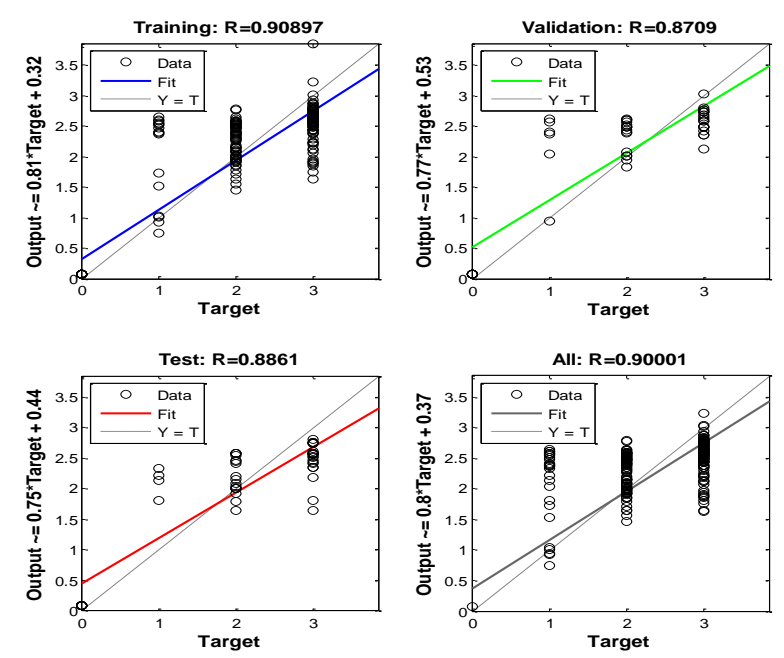

Fig 17: regression for level 3 image when using DB mother function level 5.

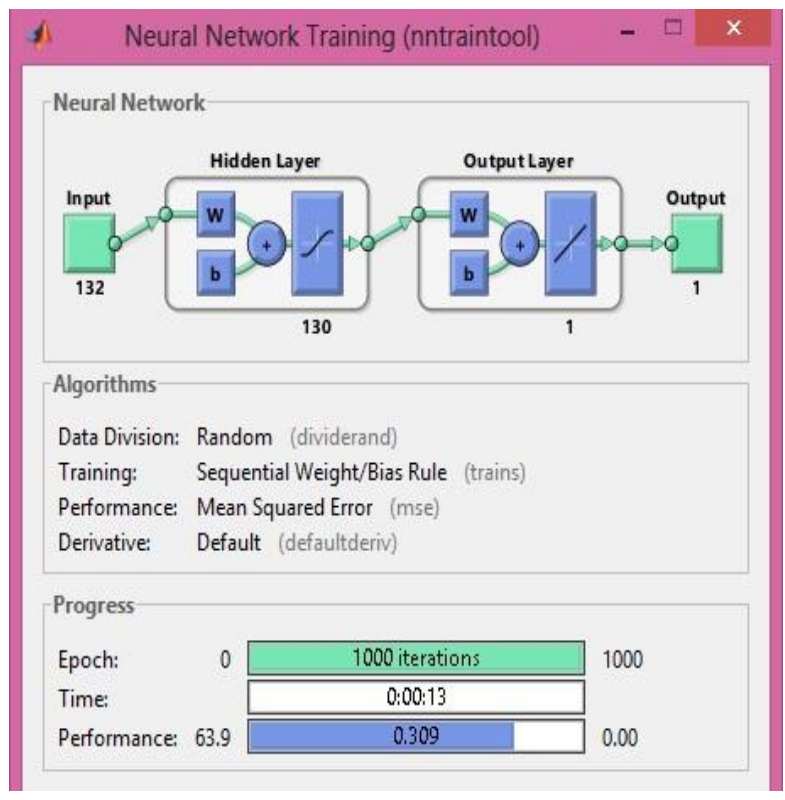

Fig 18: Neural network training interface for level 4.

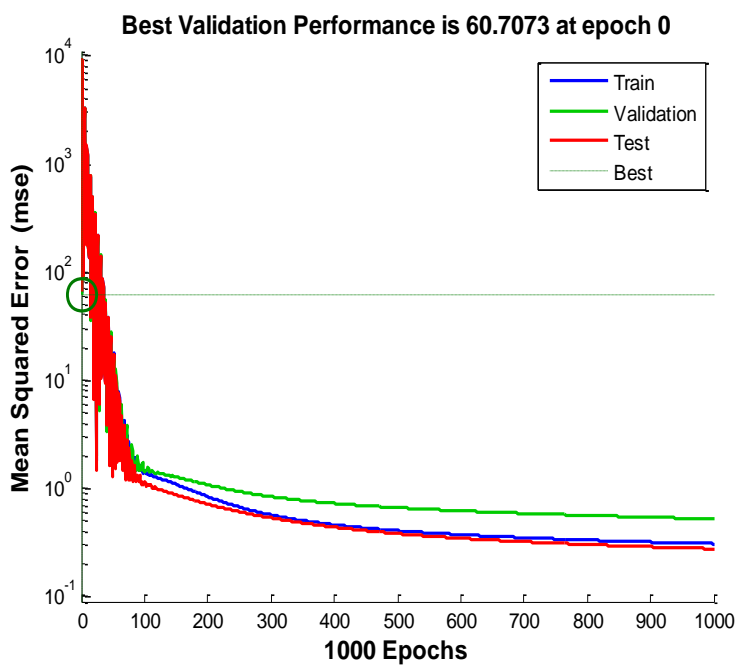

Fig. 19: Neural network training curve for level 4.
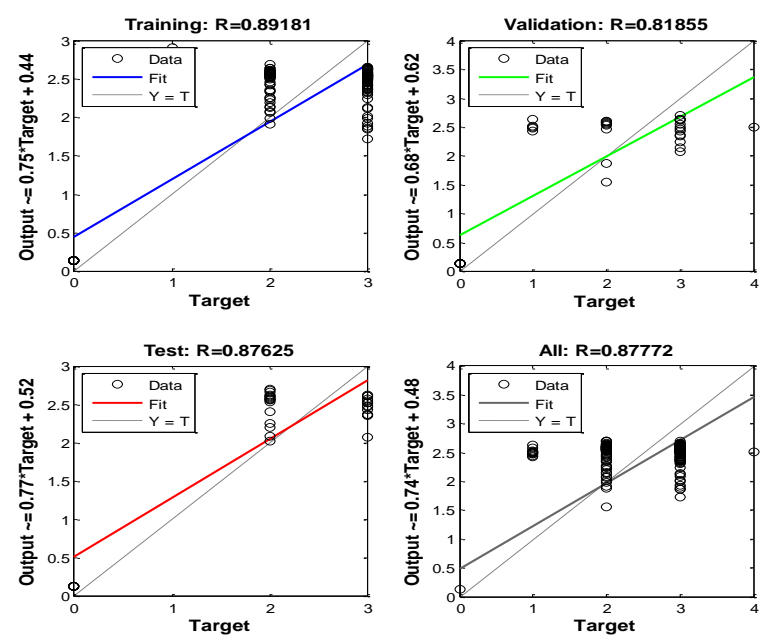

Fig. 20: regression for level 4 image when using DB mother function level 5. 


\section{REFERENCES}

[1] S. Haykin, Neural Networks, Comprehensive Foundation. NewDehli: Prentice Hall of India, 2006.

[2] A. Jemal, R. Siegel, E.Ward, Y. Hao, J. Xu, and M. J. Thun. Cancer statistics, 2009. CA Cancer J Clin, 59(4) 2009,225-249,.

[3] Ries, L. A. G., Harkins, D., Krapcho, M., Mariotto, A., Miller, B. A., Feuer, E. J., et al. (2005, Novermber, 2005). SEER Cancer Statistics Review, 1975-2003.

[4] Baffa, R., Letko, J., McClung, C., LeNoir, J., Vecchione, A., \& Gomella, L. G., Molecular genetics of bladder cancer: targets for diagnosis and therapy. J Exp Clin Cancer Res, 25(2), 2006, 145-160,

[5] Botteman, M. F., Pashos, C. L., Redaelli, A., Laskin, B., \& Hauser, R. The health economics of bladder cancer: a comprehensive review of the published literature. Pharmacoeconomics, 21(18), 2003, 1315-1330

[6] Greene, F. L., Page, D. L., Fleming, I. D., Fritz, A. G., Balch, C. M., Haller, D. G., et al. (Eds.). AJCC Cancer Staging Handbook (Sixth ed.). New York: Springer, 2002 .

[7] Rajesh, A., Sokhi, H.K., Fung, R., Mulcahy, K.A.,and Bankart M.J.G,: "Bladder cancer: Evaluation of staging accuracy using dynamic MRI", Clinical Radiology, 66, 2011, 1140-1145,

[8] Marchevsky, A. Mario," The Use of Artificial Neural Networks for the Diagnosis and Estimation of Prognosis in Cancer Patients", Chapter 9, Department of Pathology, Cedars-Sinai Medical Center, CA 90048, USA, ,2007, 243-259.

[9] Fuchs, T.,: "Computational pathology: Challenges and promises for tissue analysis", Computerized Medical Imaging and Graphics 35, 2011, 515-530,

[10] Rosenkrantz, B. Andrew , Niver, E. Benjamin, Kopec, Martin , Berkman, S.Douglas, Lepor, Herbert, . Babb , S.James and Hecht, Elizabeth M.,: "T1 hyperintensity of bladder urine at prostate MRI: frequency and comparison with urinalysis findings", Clinical Imaging 35, 2011, 203-207.
[11] Sakai, I., Miyake, H., Harada, K., Hara, I., Inoue, T. A., \& Fujisawa, M. Analysis of factors predicting intravesical recurrence of superficial transitional cell carcinoma of the bladder without concomitant carcinoma in situ. Int J Urol, 13(11), 2006. 1389-1392.

[12] G.Alizadeh, J. Frounchi, M. Baradaran Nia, S. Asgarifar, and M. H. Zarifi, "An Artificial Neural Network for Prediction of Cetane Number in Diesel Fuel Implemented on a FPGA," in Proc. IEEE International Conference on Computer and Communication Engineering, Kuala Lumpur, Malaysia, 2008.

[13] Ringner, M. and Peterson, C., : "Microarray-based cancer diagnosis with artificial neural networks", Biotechniques, 34, (Suppl 3), 2003, 30-35.

[14] 1 - Yoldaş O, Tez M, Karaca T.,: "Artificial neural networks in the diagnosis of acute appendicitis". Am J Emerg Med, 7, 2012, 1245-7.

[15] S. Natarajan, R. Singh, M. Lee, B. Cox, M. Culjat, H. Lee, and W. Grundfest, IStep-fmcw signaling and target detection for ultrasound imaging systems with conforma transducer arrays," in Proceedings of SPIE, vol. 7555, 2010.,p. 75550M.

[16] Kipp, B. R., Sebo, T. J., Griffin, M. D., Ihrke, J. M., \& Halling, K. C. Analysis of polyomavirus-infected renal transplant recipients' urine specimens: correlation of routine urine cytology, fluorescence in situ hybridization, and digital image analysis. Am J Clin Pathol, 124(6), $2005,854-861$

[17] I. Jolliffe. Principal Component Analysis. SpringerVerlag New York Inc., New York, NY, 1986.

[18] L. D. F. Costa and R. M. Cesar, Jr. Shape Analysis and Classification: Theory and Practice. CRC Press LLC, Boca Raton, FL, 2001.

[19] Illinois , Y. Peng: " Computer-Aided Histological Analysis For Prostate Cancer Diagnosis", The Faculty Of The Division Of The Biological Sciences And The Pritzker School Of Medicine In Candidacy For The Degree Of Doctor Of Philosophy Committee On Medical Physics, Chicago, June 2010, P. 67 